\title{
A Framework for Developing a Web-Based Optimization Decision Support System for Intra/Inter-organizational Decision-Making Processes
}

\author{
Andrés Boza, Angel Ortiz, and Llanos Cuenca \\ Research Centre on Production Management and Engineering (CIGIP) \\ Universidad Politécnica de Valencia. Camino de Vera s/n, 46022 Valencia, Spain \\ \{aboza, aortiz, 1lcuenca\} @cigip.upv.es
}

\begin{abstract}
The evolution in information technology has allowed the development of new DSS architectures. This paper presents a framework for developing a web-based optimization decision support system for intra/inter-organizational decision-making processes. Data exchange in inter-organizational decision, separate decision and data models, and web services technology have been raised. Considering these factors a framework for developing a DSS with web services have been defined. In a business context, these web services could be offered for different web services provider companies and used by the different participants in an intra/inter-organizational decision-making process. In order to contextualize this framework, three scenarios have been defined and different relationships have been identified between the participating enterprises.
\end{abstract}

Keywords: Decision Support Systems, optimization, web services.

\section{Introduction}

Decision Support Systems (DSS) have been used to help in a wide range of enterprise decision-making processes. The evolution in information technology has allowed the development of new DSS architectures, from stand-alone DSS to Group Decision Support Systems, OLAP and data warehouse technology in DSS, agent-based DSS or Web-based DSS [1][2][3]. Power [4] defined the expanded DSS framework with five categories: data-driven, model-driven, knowledge-driven, document-driven and communication-driven DSS.

A new generation of web-based model-driven DSS is beginning to emerge [3]. These systems take advantage of the web services concept. A single DSS problem may be solved using multiple modelling or solution paradigms developed by different sources as separate web services and the results are then presented to the user in an aggregated or summarized form. Developing a web-based model-driven DSS requires identifying its components and describing their functionalities.

This paper presents a web-based optimization DSS framework for intra and interorganizational decision-making processes in order to be used in the early design stages of a DSS. The framework is focused on service-oriented architectures (SOA) 
using web services. This paper deals with the model-driven DSS, specifically about optimizations models, and increasing their potential by means of web technology.

\section{Data Exchange in Inter-organizational Decisions}

Huxham [5] defines inter-organizational collaboration as a "process in which organizations exchange information, alter activities, share resources and enhance each other capacity for mutual benefit and a common purpose by sharing risks, responsibilities and rewards". Relationships among trading partners in a supply network have been categorized as [6]: 1) Transactional (automation of buyer-seller EDI-based transactions), 2) Information-Sharing (the partners are given access to a system that has the shared information in it, or one partner transmits shared information to the other partner), and 3) Collaborative (information is not just exchanged and transmitted, but it is also jointly developed by the trading partners working together). According to Vernadat [7] in enterprise interoperability the main challenge is operations optimization via these co-decision, co-ordination, and even negotiation mechanisms.

DSS in inter-organizational systems have been defined for SCM [8], X-networks [9], Extended Enterprise [10], Virtual Enterprise [11] or in the broad area of distributed decision making contexts [12]. European initiatives have been elaborated to develop interoperability of enterprise (decision) applications [13] and will be elaborate in future internet enterprise systems to support inter- and intra- organisational collaboration [14].

The internal/external enterprise decision structures are changing. For example, planning collaboration cannot be just a solution between close partners, but needs to be implemented with a large number of different business partners [15]. The goal today must be solutions that enable mass collaboration. In the Virtual Enterprise (VE) paradigm, enterprises must share and exchange a part of their internal local information in order to interact and cooperate with each other towards the achievement of the global VE goals [16]. A member of the VE may decide to give access to its partners, with different levels of visibility according to their cooperation agreements, to part of the information it owns [17]. About technological aspects, the major principles and technological waves that have prevailed in building integrated or interoperable enterprise systems so far have been [7]: 1) Data integration, 2) Object-oriented approaches and object request brokers (ORBs), 3) Business process modelling (BPM) and process-oriented approaches, 4) Enterprise application integration (EAI), 5) Web services and service-oriented architectures (SOAs). The architectural style of next generation technological platforms intended to support design, deployment, and execution of extended business processes inside organizational networks, must be guided by interoperability principles at the processes, services, or data level, and service-oriented architectures attempt to address these issues [18]. Web Services are a key component of the emerging, loosely coupled, web-based computing architecture. Other systems may interact with a web service in a manner prescribed by its definition, using XML based messages conveyed by Internet protocols. The web services specifications offer a communication bridge between the heterogeneous computational environments used to develop and host applications [19]. The web environment is emerging as a very important DSS development and delivery platform [2]. DSS built for this 
environment, named web-based DSS [4], use different technologies [20][21]. Among these technologies, web services are considered a viable technological platform for web-based optimization [22].

\section{DSS with Separate Decision and Data Models}

Data Modelling, Decision Modelling, and Model Analysis and Investigation, are logical constructs, which play a leading role both in the interaction of information systems and decision technologies, as well as in rational decision making [23]: 1) Data Modelling refers to the 'structured' internal representation and external presentation of recorded facts. Broadly speaking this provides the decision-maker with information about their decision problem. 2) Decision Modelling is the development of a model, or a range of models that captures the structure as well as the decisions in respect of a given problem. These models are used to evaluate possible decisions in a given problem domain, and the probable outcomes. 3) Model Analysis and Investigation refers to the instantiation of the model with data, and the evaluation of the model parameters as well as the results in order to gain confidence and insight into the model. This separation between data and models, already included in [24], has been used in multiple DSS designs [25][26][27][28]. [10] proposes a framework for a decision support system in a hierarchical extended enterprise decision context. Figure 1 illustrates, in a simplified form, some of the components defined and its relationships without include hierarchical decision details.

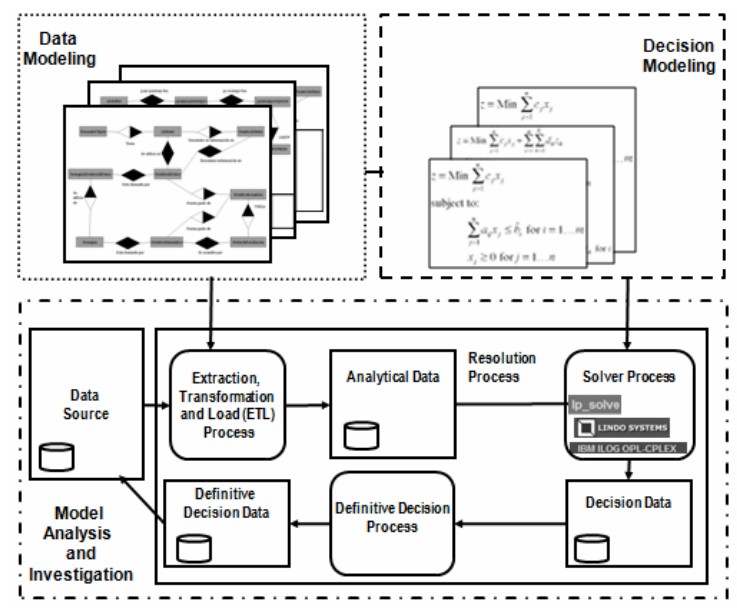

Fig. 1. Separate decision and data models (adapted from [10])

[22] shows this separation in web-based environments for optimization. A web enabled system for optimization should aim at: a) Providing each single optimisation tool as an independent service, b) Enabling remote interaction between the optimization components to provide a complete problem solving environment, c) Combining the provision of the tools with the provision of user support and security. This can be 
achieved by adopting an IT architecture based on web services, so we propose to use this technology.

\section{A Framework for Developing a DSS Using Web Services for Separate Intra/Inter-organizational Data and Decision Models}

The components have been identified and classified as Web Services, Data exchange, Roles and Organization's Data Sources (figure 2).

\section{Web Services}

Models Manager: Makes possible the definition and links of Decision Models and Data Models. Decision Models Manager: Enables the definition and storage of Decision Models. Data Models Manager: Enables the definition and storage of Decision Data Models. Model Analysis and Investigation: Makes possible the instantiation of the Decision Data Model to solve Decision Models and obtain a Definitive Decision Data. Decision Data Model Instantiation: Carries out the extraction and transform of data from Organization's Data Sources to instance a Decision Data Model with organizations' data. Solver Process: Performs the resolution process which is capable of understanding a Decision Model Instance to solve and obtain the Decision Data. Definitive Decision Process: Allows decision-makers to consider additional factors in order to make its definitive decision.

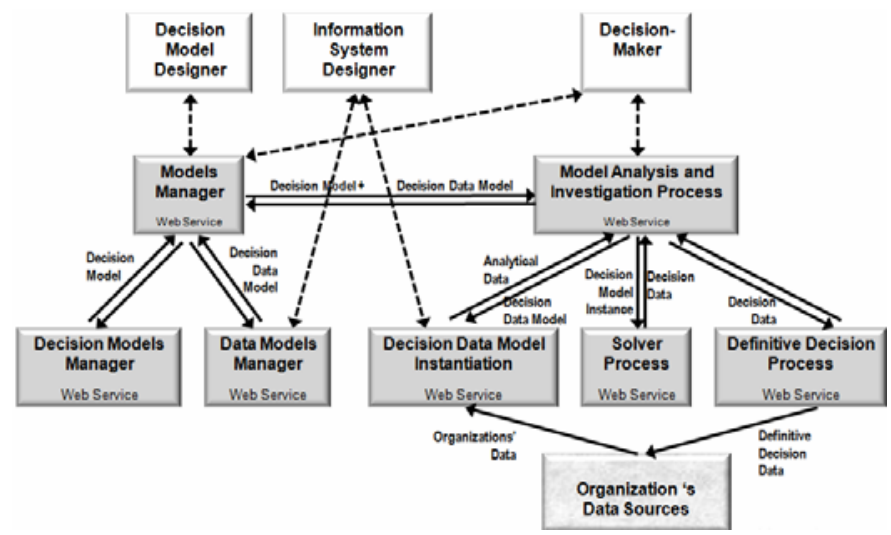

Fig. 2. A framework to developing a DSS using web services for separate intra/interorganizational data and decision models

\section{Data exchange}

Decision Model: A mathematical representation of a decision problem to find the best solution. The decision model uses components of a Decision Data Model as index, data entries, decision or bounds variables for its definition, but not their values. Decision Data Model: A formal definition and format of data, compressible by computer systems, used in decision models. These decision data models will be instanced with organizations' data for a decision model in the Model Analysis and Investigation. 
Analytical Data: Organizations' data obtained from the Organizations' Data Sources according to a Decision Data Model. Decision Model Instance: A Decision Model instanced with Analytical Data. Decision Data: Data obtained by a Solver Process with a Decision Model Instance. Definitive Decision Data: Data about the definitive decision taken by the decision-maker in a Decision Model. Decision Data obtained by the solver can be updated by the decision-maker in function of their judgments and choices.

\section{Roles}

Decision-Maker: Person(s) in charge of a decision making for a decision problem. Decision Model Designer: Person(s) in charge of constructing the suitable Decision Models. Information System Designer: Person(s) in charge of constructing the suitable Information System to give service to the information necessities

\section{Organization's Data Sources}

Data bases, files or spreadsheets in organization information systems where the data of a Decision Data Model are located.

\section{The Proposed Framework in Three Scenarios}

In order to contextualize the framework in an intra/inter-organizational environment, three scenarios has been defined (figure 3): Scenario 1: Intra-organizational data and decision model. There is not information or decision model sharing between organizations. Scenario 2: Inter-organizational data and intra-organizational decision model. There exists information sharing between organizations but not a joint decision model. Scenario 3: Inter-organizational data and decision model. There is information sharing and joint decision model between organizations.

\subsection{Intra-organizational Data and Decision Model}

In this context, Decision Model Designers and Decision-Makers interact with the Model Manager Web Service in order to define Decision Models and Decision Data Models, which will be later instanced. Defining separate Decision Models and Decision Data Models allows Decision Model Designer and Decision-Makers to define some Decision Models that use a same Decision Data Model.

Information System Designer interacts with the Data Models Manager web service and Decision Data Model Instantiation web service in order to define the instantiation process to obtain Analytical Data. Decision-Maker use Model Analysis and Investigation web service in order to instance a Decision Model with a data set, resolve it and make a decision. In this sense, Decision-Makers could resolve different previously defined Decision Models, and these could be instanced with different data sets (different companies, departments or simulations data) that fit with its Decision Data Model.

It is possible to implement a DSS platform in the organization with all these web services, but using the web services technology facilitates that they can be provided by web services provider companies. In this sense, a web services provider company 
with mathematical model specialized staff could advice on model definitions and host the defined models, another provider could offer web services for data management and a third could provide solver web services. Therefore, the main advantage of the framework in this scenario for the organizations is reuse decision models, and from a technological perspective, the main advantage is to build a DSS with components provided for different web services companies.

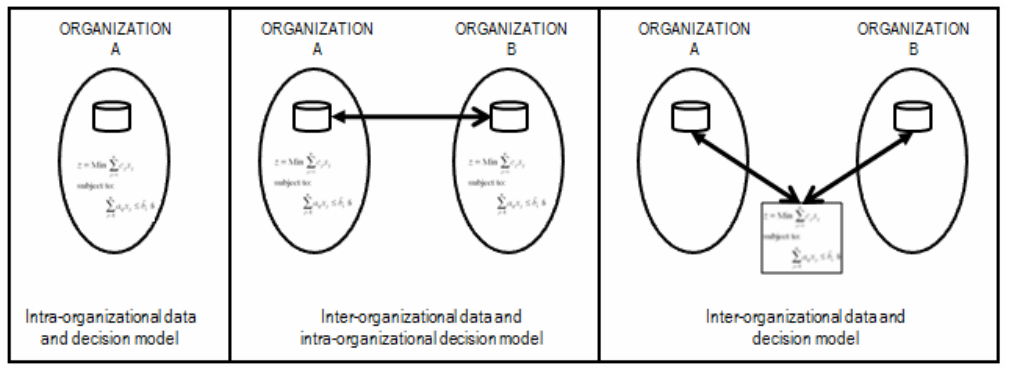

Fig. 3. Three inter/intra-organizational DSS scenarios

\subsection{Inter-organizational Data and Intra-organizational Decision Model}

In this scenario, there is information-sharing between organizations but not joint decision models. Besides the features included in the previous scenario, additional features can be identified: Participating organizations must work jointly in the data model definition that each organization shares with the other. Also, it is necessary to define a Decision Data Model Instantiation in order to retrieve data, not for its DSS but for the partner organization. In these sense, the participating organization require establishing the data exchange periodicity in order to orchestrate the web services.

An advantage in this case is to use web services technology to exchange data between DSS web-based or uses a common data-manage web services provider company.

\subsection{Inter-organizational Data and Decision Model}

There are decision models defined jointly by the participating organization and information sharing. Besides the features included in the previous scenarios, additional features can be identified:

In this scenario, the Models Manager web service is a common tool to jointly define share Decision Models and Decision Data Models. Each organization must define its Decision Data Model Instantiation web service in order to retrieve its data subset that participates in the joint Decision Data Model. The participating organizations require establishing a common schedule in order to resolve its share Decision Models and the necessary mechanisms to define a definitive decision in function of the (interorganizational) decision-makers judgments and choices.

A DSS developed from the framework for this scenario allows defining decision models, share data and solve shared decisions with a web-based technology to be used in intra/inter-organizations decision-making processes. 


\section{Conclusions}

DSS for intra and inter-organizational decision have been defined in a wide range of decision making situations. The technological advances in the Internet make available new DSS advances. In this sense, service-oriented architectures using web services allow defining new web-based DSS. The proposed framework for developing DSS using web services for separate intra/inter-organizational data and decision models draws on web technology to design Decision Models and Decision Data Models which will be later instanced and solved to make a decision. Its components have been identified and classified as web services, data exchange, roles and organization's data sources. Designing a DSS with web services allows splitting its functionality using these web services. Different web services providers can offer these and can be used by the different participants in an intra/inter-organizational decision-making process. Contextualizing the proposed framework using three scenarios has allowed identifying different aspects of the proposal with the participating enterprises in an intra/inter-organizational decision process and web services providers companies.

\section{References}

1. Carlsson, C., Turban, E.: DSS: directions for the next decade. DSS 33, 105-110 (2002)

2. Shim, J.P., Warkentin, M., Courtney, J.F., Power, D.J., Sharda, R., Carlsson, C.: Past, present, and future of decision support technology. DSS 33, 111-126 (2002)

3. Power, D.J., Sharda, R.: Model-driven decision support systems: Concepts and research directions. DSS 43, 1044-1061 (2007)

4. Power, D.J.: Supporting Decision-Makers: An Expanded Framework. In: Informing Science Conference, Krawkow, Polonia, June 19-22 (2001)

5. Huxham, C.: Creating Collaborative Advantage. Sage Publishers, London (1996)

6. AMR: Are we moving from buyers and sellers to collaborators? SCM Report. American Manufacturing Research Inc. (July 1998)

7. Vernadat, F.B.: Interoperable enterprise systems: Principles, concepts, and methods. Annuals Reviews in Control 31, 137-145 (2007)

8. Julka, N., Srinivasan, R., Karimi, I.: Agent-based supply chain management-1: framework. Computers and Chemical Engineering 26, 1755-1769 (2002)

9. Alix, T., Zolghadri, M., Bourrieres, J.P.: A DSS for production and procurement planning of enterprises X-networks. In: IEEE Int. Conf. on Systems, Manufacturing and Cybernetics (2004)

10. Boza, A., Ortiz, A., Vicens, E., Poler, R.: A Framework for a DSS in a Hierarchical Extended Enterprise Decision Context. LNBIP, vol. 38, pp. 113-124 (2009)

11. Zhou, Q., Ristic, M., Besant, C.B.: An Information Management Architecture for Production Planning in a Virtual Enterprise. IJAMT 16, 909-916 (2000)

12. Shneeweiss, C.: Distributed decision making-—a unified approach. European Journal of Operational Research 150, 237-252 (2003)

13. Chen, D., Doumeingts, G.: European initiatives to develop interoperability of enterprise applications-basic concepts, framework and roadmap. Annual Reviews in Control 27, 153 162 (2003)

14. FInES: Future Internet Enterprise Systems Cluster. Position Paper, Version 3.0 (2009) 
15. Holmström, J., Främling, K., Kaipia, R., Saranen, J.: Collaborative planning forecasting and replenishment: new solutions needed for mass collaboration. Supply Chain Management: An International Journal 7(3), 136-145 (2002)

16. Frenkel, A., Afsarmanesh, H., Garita, C., Hertzberger, L.O.: Supporting information access rights and visibility levels in virtual enterprises. In: IFIP Conf. on Infrastructures for Virtual Organisations (2001)

17. Camarinha-Matos, L.M., Pantoja-Lima, C.: Cooperation coordination in virtual enterprises. Journal of Intelligent Manufacturing 12, 133-150 (2001)

18. Franco, R.D., Ortiz, A., Lario, F.C.: Modeling extended manufacturing processes with service-oriented entities. Service Business 3, 31-50 (2009)

19. W3C, Web Services Choreography Description Language Version 1.0 (2005)

20. Power, D.J., Kaparthi, S.: Building Web-based decision support systems. Studies in Informatics and Control 11(4), 291-302 (2002)

21. Bhargava, H.K., Power, D.J., Sun, D.: Progress in Web-based decision support technologies. Decision Support Systems 43, 1083-1095 (2007)

22. Valente, P., Mitra, G.: The evolution of web-based optimisation: From ASP to e-Services. DSS 43, 1096-1116 (2007)

23. Dominguez-Ballesteros, B., Mitra, G., Lucas, C., Koutsoukis, N.-S.: Modelling and solving environments of mathematical programming (MP): a status review and new directions. Journal of the Operation Research Society 53, 1072-1092 (2002)

24. Geoffrion, A.M.: An introduction to structured modelling. Management Science 33(5), 547-588 (1987)

25. Dolk, D.R.: Integrated model management in the data warehouse era. European Journal of Operation Research 122, 198-218 (2000)

26. Lee, K.W., Huh, S.Y.: A model-solver integration framework for autonomous and intelligent model solution. DSS 42, 926-944 (2006)

27. Liew, A., Sundaram, D.: Flexible modelling and support of interrelated decision. DSS 46, 786-802 (2009)

28. Liu, S.F., Duffy, A.H.B., Whitfield, R.I., Boyle, I.: Integration of decision support systems to improve decision support performance. In: Knowledge and Information System (2009) 